\title{
Summary of the LARP Mini-Workshop on Electron Lens Simulations at BNL
}

A. Valishev (FNAL), Y. Luo, W. Fischer (BNL)

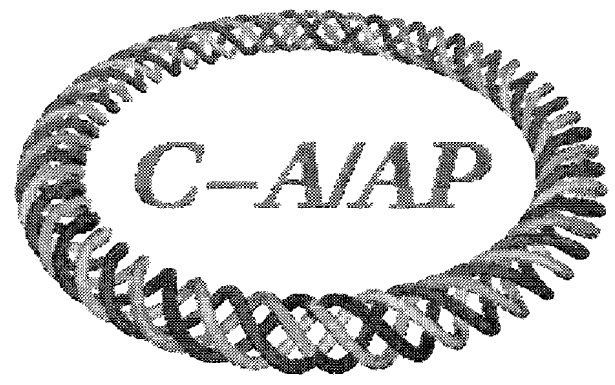

\section{Collider-Accelerator Department Brookhaven National Laboratory Upton, NY 11973}

Notice: This document has been authorized by employ ees of Brookhaven Science Associates, LLC under Contract No. DE-AC02-98CH10886 with the U.S. Department of Energy. The United States Government retains a nonexclusive, paid-up, irrevocable, world-wide license to publish or reproduce the published form of this document, or allow others to do so, for United States Government purposes. 


\title{
Summary of the LARP Mini-Workshop on Electron Lens Simulations at BNL
}

\author{
A. Valishev (FNAL), Y. Luo, and W. Fischer (BNL)
}

December 24,2008

\section{Introduction}

This was a 1 day workshop that brought together a group of people working on beam-beam simulations, in particular those comprising the simulation part of LARP Beam-Beam Task. There were 8 participants from BNL, and 3 from FNAL:

\author{
Natalia Abreu, BNL \\ Joanne Beebe-Wang, BNL \\ Ricardo DeMaria, BNL \\ Wolfram Fischer, BNL \\ Hyung-Jin Kim, FNAL
}

\author{
Yun Luo, BNL \\ Ryoichi Miyamoto, BNL \\ Christoph Montag, BNL \\ Guillaume Robert-Demolaize, BNL \\ Tanaji Sen, FNAL \\ Alexander Valishev, FNAL
}

A notable absence was Andreas Kabel from SLAC.

The goals of the mini-workshop were:

1. To identify the beam-beam effects in LHC and RHIC that could be mitigated using electron lenses, and to define machine and beam parameters one should be looking at in simulations.

2. To assess the group capabilities and establish means of collaboration.

3. To establish near and long term simulation program, set priorities and a schedule.

A list of questions was assembled as a basis for the discussions:

- How can the luminosity gain from an electron lens be estimated?

- What can we conclude from short-term measures? (footprints, tuned diffusion, Lyapunov exponents) 
- What can we conclude from long-term measures? (dynamic aperture, emittance growth beam lifetime)

- How do we benchmark simulations?

- Electron lenses in BNL simulations stabilize particles below 3 sigma, but reduce stability above 4 sigma. Why? (phase advance)

- What are the sensitive parameters? (phase advance? current noise? shape? shape noise? position noise?)

- What are the insensitive parameters?

- Which parameters were found to be sensitive/insensitive during Tevatron electron lens operation?

- What effects need to be included in simulations? (magnetic field errors in IRs, magnetic field errors in arcs, tune ripple)

- Do we need to consider the finite electron beam rigidity? (Burov PRST-AB paper)

- How do we treat crossing strong resonances in simulations (when beams go into collisions)?

- How are Tevatron, RHIC, LHC different?

- How are LIFETRAC, BBSIM, SixTrack, PlibB different?

- Where do we get enough CPU power for the simulations?

- Can we calculate resonance driving terms without and with an electron lens?

Presentations are available at

http://larpdocs. fnal.gov/LARP-public/DocDB/DisplayMeeting?conferenceid $=64$

We tried to concentrate on discussions and kept the amount of presentations minimal. The agenda for the day was divided into 3 parts:

1. Physics questions (to discuss the effects we would be looking at)

2. Physics tools (what can be learned from simulations, how one interprets results)

3. Simulation tools (what tools do we currently have on hand, what can be done, communication, etc.) 


\section{Summary of electron lens physics}

1. Tevatron experience suggests that the combination of head-on and long-range effects is important, and the beams separation of $>6$ sigma must be maintained.

2. Both Tevatron and RHIC show the importance of chromatic effects (tune chromaticity, chromatic beta-beat).

3. In RHIC simulations higher order nonlinearities in the triplets have a strong impact on the beam dynamics. The $3 Q \times$ resonance is important at the current proton working point.

4. The operational experience with Tevatron Electron Lenses was summarized. The critical parameters are: transverse beam alignment to $0.2-0.5 \mathrm{~mm}$, current ripple $<0.1 \%$, pulse stability $<1 \mathrm{~ns}$, limited dispersion at the electron lens.

5. Beam-Beam effects at LHC. Under the LPA upgrade scenario the bunch population may reach $5 \times 10^{11}$, hence the head-on effect can become an issue. Given the presence of long-range collisions this provides vast field for beam-beam effect compensation, both head-on and long-range.

6. RHIC has nominally no long-range effects, and the head-on effect is not yet as strong as in the Tevatron (total beam-beam induced tune spread reached 0.012 in RHIC and up to 0.03 in the Tevatron. Beam-beam effects do limit the RHIC luminosity already. With the upgrade it may be possible to attain the beam-beam parameter higher that at the Tevatron. Installation of an electron lens at RHIC is also envisaged as a test bed for eRHIC. An electron lens may also ameliorate the beam-beam effect on the electrons in the ring-ring version of eRHIC as was shown by $\mathrm{C}$. Montag.

\section{Summary of Physics Tools}

1. Short-term measures include: tune footprint, diffusion coefficients, tune diffusion, resonance driving terms, and short-term dynamic aperture. Short-term here means typically thousands of turns. The advantage of using these tools is in computational speed. However, interpretation of the results is not straightforward. Specifically, it is unclear how one correlates the short term measures to macroscopic beam parameters like life time. We agreed that many of these measures contain valuable information but no single short-term indicator is a reliable long-term predictor.

2. With the above being said, we will mostly rely on long-term tracking simulations to draw conclusions. Long-term here means at least minutes, sometimes hours of store time. The main parameters for evaluation are beam life time and emittance growth. For head-on beam-beam problems the dynamic aperture is an unreliable measure since it evaluates the stability at large amplitudes where the beambeam force is weak. In the BNL simulations so far it was also found that emittance growth is too noisy a signal to be useful for the comparison of different cases.

\section{Available Computing Tools}

1. A number of people have worked on short-term simulations: Yun has calculated tune footprints, tune diffusion, Lyapunov exponents and dynamic apertures. Tanaji and Hyung-Jin at FNAL, and Natalia at BNL 
have evaluated diffusion coefficient (which can were also used to assess long-term behavior). The codes they use are BBSIM and SixTrack, respectively. It is advantageous that both codes aiso provide long-term tracking capabilities so a direct comparison is possible. Yun has

2. The long-term codes are: BBSIM, LIFETRAC, PlibB, and SixTrack. They have many similarities - weakstrong macro particle tracking with Gaussian strong bunch (with exception of PlibB that has also strongstrong option). SixTrack uses element-by-element tracking and models can include magnetic field errors in the lattice, the rest represent the machine lattice with linear 6D maps and thin nonlinear elements. All codes include chromatic effects.

3. At FNAL, the two codes (LIFETRAC and BBSIM) share a 16-node $x$ 4CPU cluster. A typical run of about 10,000 particles over $10^{7}$ turns takes 3-7 days to complete depending on the accelerator configuration. Similar performance is observed when using SixTrack. The group at BNL is currently using a small cluster and planned to use the LHC@HOME facility (http://hcathome.cern.ch/). However, installation of the special SixTrack version needed for the electron lens simulation was held up, and user accounts were now obtained for a BlueGene computer at BNL, and a NERSC cluster at LBNL.

\section{The Near Term Plan}

We defined a benchmark exercises for code. A RHIC lattice and beam (specified by Yun) and an LHC lattice and beam (specified by Sasha) should be exercises with for 4 cases:

No beam-beam, with beam-beam, with half beam-beam compensation, with full beam-beam compensation

For all 4 cases 3 conditions can be examined

- Follow a single particle at 1 sigma over 10000 turns (must not be chaotic)

- Follow a few particles over 10000 turns

- Calculated lifetime and emittance growth of about 5000 particles over 10 million turns.

The lattice and beam specification for both RHIC and the LHC must include apertures and the number of slices for the beam-beam interaction.

We will create a web page for each case to put machine lattices, beam parameters, etc. The natural way for exchanging results is the LARP document database

\section{The Long Term Plan}

To assess the robustness of the head-on beam-beam compensation by the electron lens, a number of parameters need to be varied. Among these must be the parameters found to be sensitive in the operation of the Tevatron electron lens. The list of all parameter under consideration includes: phase advance between beam-beam interaction and electron lens, electron position and position noise, 
electron lens profile and profile noise, electron lens strength (current) and strength noise, dispersion at the electron lens. Codes must also be benchmarked against experimental data. 